\title{
Tendances de l'immigration au Paraguay. Premiers résultats du Recensement 2002
}

Sylvain Souchaud

\section{(2) OpenEdition \\ 12 Journals}

Édition électronique

URL : https://journals.openedition.org/remi/2037

DOI : $10.4000 /$ remi.2037

ISSN : $1777-5418$

Éditeur

Université de Poitiers

\section{Édition imprimée}

Date de publication : 1 décembre 2004

Pagination : $179-184$

ISBN : 2-911627-38-5

ISSN : 0765-0752

Référence électronique

Sylvain Souchaud, "Tendances de l'immigration au Paraguay. Premiers résultats du Recensement 2002 ", Revue européenne des migrations internationales [En ligne], vol. 20 - n³ | 2004, mis en ligne le 25 septembre 2008, consulté le 14 avril 2022. URL : http://journals.openedition.org/remi/2037 ; DOI : https://doi.org/10.4000/remi.2037

Ce document a été généré automatiquement le 14 avril 2022.

(c) Université de Poitiers 


\title{
Tendances de l'immigration au Paraguay. Premiers résultats du Recensement 2002
}

\author{
Sylvain Souchaud
}

1 En 2002, le Paraguay organisait, dix années après le précédent, un recensement de sa population. Les résultats produits depuis lors l'ont été à l'échelle des 14 départements que compte le pays (Dirección General de Estadísticas, 2003). Récemment, certaines données analysées au niveau des 218 districts ont été diffusées (Dirección General de Estadísticas, 2004), organisées autour de trois axes: la structure par âge de la population, la population étrangère et l'habitat. Pour l'ensemble des variables, populations rurales et urbaines sont distinguées.

2 Nous nous limiterons ici à l'examen des données sur la population étrangère ${ }^{1}$. Bien que partielles, elles nous permettent de faire un point sur la situation du Paraguay dont le profil migratoire actuel s'est construit dans les dernières décennies, et d'examiner en particulier l'évolution de la colonisation brésilienne dans l'extrême est du territoire, phénomène qui nous avait intéressé à la fin des années quatre-vingt-dix (Souchaud, 2002).

3 La finesse apportée par le changement d'échelle d'analyse, dans un pays où les évolutions socio-démographiques sont marquées par une forte hétérogénéité spatiale, ainsi que la prise en compte de la thématique particulière des populations immigrées nous ont semblé constituer une nouveauté suffisante pour porter un premier regard, certes très incomplet, sur ces résultats.

\section{Le Paraguay : pays d'émigration et d'immigration frontalière}

4 L'histoire migratoire paraguayenne est avant tout celle d'un pays d'émigration. L'expatriation de sa population, essentiellement à destination des pays voisins et 
notamment l'Argentine, est une constante plus ou moins marquée selon les époques. Mais, dans la deuxième moitié du $\mathrm{XX}^{\mathrm{e}}$ siècle, le Paraguay devient également un pays d'immigration. Contrairement à ses voisins du Cône Sud, l'Argentine, le Brésil et l'Uruguay (avec lequel le Paraguay ne partage pas de frontière), le Paraguay n'a que très peu connu les courants d'immigration européenne des XIX ${ }^{\mathrm{e}}$ et $\mathrm{XX}^{\mathrm{e}}$ siècles (Pidoux de Drachenberg, 1975). L'attractivité du territoire paraguayen, très faible jusque-là, évolue dans les années soixante-dix, lorsque s'active l'immigration brésilienne. Aux recensements la population née à l'étranger représente $3,4 \%$ de la population totale en 1972, 5,6\% en 1982 et 4,6\% en 1992. Le fort croît démographique sur la période implique une variation de la part de la population immigrée dans la population totale, néanmoins, le nombre des individus nés à l'étranger augmente fortement et constamment, passant de 81100 en 1972 à 190907 en 1992. En 2002, la situation évolue à nouveau puisqu'on constate la baisse relative du nombre des personnes nées à l'étranger; elles représentent désormais $3,4 \%$ de la population totale, ralentissement de la tendance antérieure qui se double d'une baisse de l'effectif, lequel atteint 173852 individus en 2002.

En 2002, les immigrants recensés sont pour l'essentiel brésiliens, près de la moitié de l'ensemble, et argentins, plus du tiers (tableau 1). En dix ans l'écart entre les deux groupes s'est nettement resserré. Les effectifs brésiliens se sont réduits, passant de 108 000 à 83000 tandis que ceux nés en Argentine se sont nettement renforcés, passant de 49000 à 63 000. Comment interpréter la croissance de la population née en Argentine alors que l'attrait du territoire paraguayen semble limitée pour les Argentins? Doit-on voir les effets de la crise argentine? S'agit-il d'une migration de retour, c'est-à-dire l'installation au Paraguay d'enfants de migrants paraguayens nés en Argentine et ayant acquis la nationalité de ce pays? Quant à la baisse des effectifs brésiliens, elle ne peut s'expliquer par une procédure massive de naturalisations puisque les données fournies font état du lieu de naissance des individus. Elle signalerait par conséquent le retour des Brésiliens dans leur pays entre les deux recensements. Mais comment interpréter celui-ci ? Est-il la conséquence d'une meilleure attractivité du secteur agricole brésilien dans les années quatre-vingt-dix ? Du durcissement des relations entre Paraguayens et Brésiliens et d'un rejet de ces derniers dont un nombre important se trouve en situation de résidence et/ou d'exploitation illégale? Ou encore pour des raisons strictement foncières, sont-ce les effets combinés d'une saturation relative de l'espace de colonisation et d'une tendance à l'uniformisation agricole vers un modèle productif peu peuplant: la culture intensive motorisée implique en effet des densités rurales moindres que l'agriculture manuelle?

6 Bien que les effectifs des deux populations tendent à s'équilibrer, la répartition entre les milieux rural et urbain distingue immigrants brésiliens et argentins. Les premiers sont en majorité des ruraux alors que les seconds vivent le plus souvent dans les villes (tableau 1). En revanche leurs structures par sexes sont plus proches, avec des hommes un peu plus nombreux que les femmes, la sur-masculinité étant un peu plus accentuée chez les immigrants brésiliens.

Tableau 1 : Immigrants* brésiliens et argentins au Paraguay, 2002

\begin{tabular}{|l|l|l|l|}
\hline & Ensemble des immigrants & dont imm. brésiliens & dont imm. argentins \\
\hline
\end{tabular}




\begin{tabular}{|l|l|l|l|}
\hline \multicolumn{2}{|l|}{ Effectifs } & 82937 & 62839 \\
\hline Nombre & 173852 & 47,7 & 36,6 \\
\hline \hline Pourcentage & 100,0 & 53,1 & \\
\hline Structure par sexe (en \%) & 46,9 & 59,6 \\
\hline Hommes & 52,3 & \multicolumn{3}{l|}{} \\
\hline Femmes & 47,7 & 63,9 & 50,4 \\
\hline Distribution par milieu d'habitat (en \%) & 23,2 \\
\hline Ruraux & 42,4 & 36,1 & 76,8 \\
\hline Urbains & 57,6 &
\end{tabular}

* Nés à l'étranger.

Source : Paraguay, recensement de population, 2002.

\section{Les données en 2002 cartographiées au niveau des districts}

7 Pour présenter la répartition spatiale de l'immigration recensée en 2002, nous avons construit une carte à partir de deux données. En cercles proportionnels figurent les effectifs de population née à l'étranger et, en aplats plus ou moins grisés, la part des mêmes effectifs dans la population totale des districts. La méthode de discrétisation adoptée, qui fixe les bornes de chaque classe, correspond à une répartition par quantile. La discrétisation par quartile est affinée aux extrêmes par la présentation, des cinq premiers percentiles au bas de l'échelle et des cinq derniers à l'opposé. Par conséquent, dans la moitié des districts, la population née à l'étranger et recensée en 2002 constitue entre 0,8 et $3,1 \%$ de la population totale.

8 Étant donné l'irrégularité du maillage administratif au Paraguay, on doit combiner la lecture des deux données pour préciser l'examen et apprécier la répartition de la population née à l'étranger. Ainsi, dans le Chaco, c'est-à-dire les trois vastes départements de l'ouest, les taches sombres signalent l'importance de la population née à l'étranger. À l'inverse, les cercles nous indiquent l'insignifiance des effectifs correspondants. La combinaison des deux informations graphiques révèle le très faible poids démographique de la région occidentale et en somme, établit que le Chaco est, en quelque sorte, un semi désert humain peuplé d'immigrants.

9 L'examen de la carte montre, en premier lieu, la très inégale répartition des immigrants au Paraguay. Deux foyers principaux apparaissent: l'aire métropolitaine d'Asunción et, de manière plus diffuse, l'extrême est du pays, aux frontières brésilienne et argentine, espace qui correspond à la zone de colonisation brésilienne. 


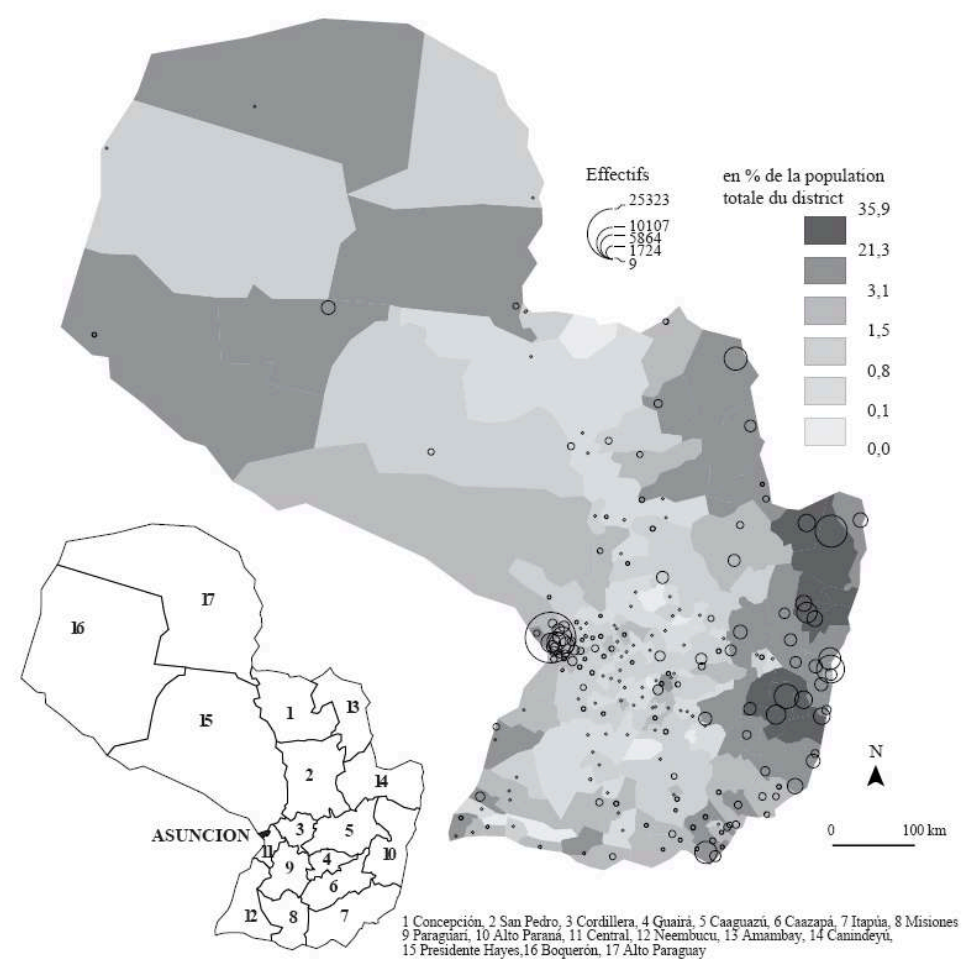

Source : DGEEC, «Indicadores por distritos - Censo 2002 », Dgeec, Assunción. Fait avec Philcarto - http://perso.club-internet.fr/philgeo [discrétisation 'Q6']. Souchaud, 2004.

10 À l'est, l'immigration est à la fois urbaine et rurale. Ainsi, des cercles importants correspondent aux districts dont le chef lieu est capitale de département. Ces villes, frontalières, captent probablement l'essentiel de l'immigration; elles se nomment Pedro Juan Caballero (département de l'Amambay), au nord, Ciudad del Este (Alto Paraná), au centre, ou encore Encarnación (Itapúa), au sud. Mais l'immigration est aussi rurale, on peut tout au moins le supposer, puisqu'à l'intérieur des terres, notamment dans le Canindeyú et l'Alto Paraná, des districts à forte composante rurale connaissent d'importants effectifs nés à l'étranger. Par ailleurs, l'immigration dans la frange frontalière est d'autant plus marquante qu'elle associe forts effectifs et importante participation à la population du district, atteignant sur un vaste espace, entre 21,3 et $35,9 \%$ de la population; tel n'est pas le cas dans la région d'Asunción où le poids démographique des immigrants reste limité.

Comment interpréter ces deux schémas spatiaux d'immigration? Peut-on établir des relations avec la répartition par pays d'origine, avec d'un côté une immigration argentine essentiellement urbaine et installée dans le centre du pays et, de l'autre, une immigration brésilienne, majoritairement rurale et située dans l'extrême est? Un tel dispositif spatial serait dans la continuité de ce que nous avions observé dans la deuxième moitié des années quatre-vingt-dix: l'immigration argentine est traditionnellement plus urbaine et vise les principaux foyers, et en particulier la capitale ; ceci est en partie confirmé par les chiffres globaux de l'immigration en 2002 qui établissent que $76,8 \%$ des immigrés argentins sont citadins. Nous ne disposons malheureusement pas encore des données 2002 de la présence immigrées par 
nationalité et par district pour étayer ces suppositions et ce point demande confirmation et approfondissement. Mais le recensement ne pourra pas éclaircir à lui seul la question, il faudrait également suivre année après année le profil des immigrants brésiliens, car il est possible que les migrations internationales urbainurbain soient de plus en plus importantes, y compris chez les Brésiliens. Ce qui irait dans le sens d'une tendance nationale, selon laquelle, entre les deux recensements de 1992 et 2002, 42 \% des migrations ont eu lieu de l'urbain vers l'urbain.

Les contours du territoire dessiné par la population immigrée en 2002 correspondent assez fidèlement à la carte de la zone pionnière brésilienne établie en 1998. Cela porterait à penser que le front de colonisation a relativement peu progressé, ou tout au moins que sa progression n'a pas été alimentée par un afflux important de population née à l'étranger, et donc n'est pas lisible sur la carte. Suivant cette hypothèse, deux schémas, qui peuvent se combiner, sont alors envisageables. Le front de colonisation peut avancer sous l'impulsion de populations paraguayennes. Rappelons à ce sujet qu'au Paraguay l'attribution de la nationalité relève du droit du sol et par conséquent, que les enfants des colons brésiliens d'hier sont aujourd'hui pour une part paraguayens. Par ailleurs, l'occupation de l'espace ne se fait pas nécessairement par l'implantation d'importants effectifs de population. Ainsi, l'agriculture moderne associe généralement faibles densités démographiques et forte emprise territoriale. Or la modernisation agricole est largement engagée au Paraguay extrême oriental, justement sous l'impulsion des Brésiliens.

13 Ce qui semble se confirmer en revanche, c'est une forte dynamique démo-spatiale à l'intérieur de la zone de colonisation, en particulier dans le département de Canindeyú dont l'importance de la population immigrée augmente et qui connaît de surcroît une forte croissance démographique, de $3.1 \%$ par an en moyenne entre 1992 et 2002, contre $2,2 \%$ sur l'ensemble du pays.

\section{Perspectives}

Doit-on interpréter ces données dans le sens d'une retombée de l'immigration au Paraguay: l'immigration des années 1972 à 1992 n'était-elle qu'une parenthèse historique, liée à une dynamique strictement brésilienne ? De la même manière que signifie la croissance de la population née en Argentine ? Il est encore impossible de le dire, et il probable que les compléments du recensement ne permettront que de modestes avancées ; les tendances sont à la fois trop récentes et trop ténues.

Par ailleurs, pour l'ensemble des observations, on doit tenir compte du sousenregistrement des mouvements migratoires. Les difficultés habituelles du traitement statistique du phénomène sont au Paraguay renforcées, d'un côté par les particularités $\mathrm{du}$ territoire national, dont les frontières sont perméables, et de l'autre par les caractères spécifiques de la migration brésilienne, très fluide et instable. Selon le recensement de 1992, le nombre d'immigrants brésiliens s'élevait à 108526 individus. Plus vraisemblablement, ce nombre atteignait, selon les estimations d'autres sources officielles, dont par exemple le Ministère des Affaires Étrangères brésilien, entre 300 000 et 350000 individus. L'immigration brésilienne se déploie dans un espace frontalier dont l'intégration, encore récente, est incomplète. Les migrants sont pour une grande part des populations en situation irrégulière. Ainsi, à la fin des années quatre-vingt-dix, nous avions observé, dans certains districts du Canindeyú, l'importance croissante 
d'une population migrante jeune, originaire de l'état voisin du Paraná, qui gagnait les petits centres urbains pour des séjours de durée relativement courte (va-et-vient éventuels). Cette forme de migration contrastait avec l'immigration de plus longue durée des cultivateurs (quoique ne nous trompons pas sur l'ancrage des agriculteurs brésiliens : les colons se caractérisent par leur forte mobilité).

À la fin des années quatre-vingt-dix le très fort poids démographique de l'immigration brésilienne dans ses espaces d'installation lui conférait, entre autres, un très forte visibilité. Il faudrait aujourd'hui envisager l'impact de la colonisation brésilienne non plus seulement par le biais de la population née à l'étranger mais en s'intéressant également aux foyers issus de l'immigration, population qui n'est pas identifiée par le recensement, afin de voir quels processus d'intégration sont éventuellement à l'œuvre. Aujourd'hui, étant donné que le poids de chacune des communautés brésilienne et argentine tend à se rapprocher, il serait intéressant d'examiner comment évolue, au sein de la société d'accueil, la perception de chacune d'entre elles.

\section{BIBLIOGRAPHIE}

Dirección General de ESTADÍSTICAS, Encuestas y Censos (2003) Principales resultados del Censo 2002, Vivienda y población, Asunción, DGEEC, www.dgeec.gov.py, 71.

Dirección General de ESTADÍSTICAS, Encuestas y Censos (2004) Indicadores por distritos, Censo 2002, Resultados preliminares, Asunción, DGEEC, www.dgeec.gov.py, 116.

PIDOUX DE DRACHENBERG Lyra (1975) Inmigración y colonización en el Paraguay, 1870-1970, Revista Paraguaya de Sociología, 12 (34), Asunción, Centro Paraguayo de Estudios Sociológicos (CEPES), pp. 65-123.

SOUCHAUD Sylvain (2002) Pionniers brésiliens au Paraguay, Paris, Khartala, 406 p.

\section{NOTES}

1. "Cuadro 2 - Paraguay: Población paraguaya y extranjera por tenencia de cédula de identidad, asistencia escolar, nivel educativo y promedio de años de estudio, según departamento área urbana-rural y distrito 2002."

INDEX

Mots-clés : population, recensement, statistiques 
AUTEUR

SYLVAIN SOUCHAUD

Géographe, chargé de recherche à l'IRD (ex ORSTOM), Tarija (Bolivie), souchaud@ird.fr 